\title{
BRECHAS DE EQUIDAD EN EL ACCESO A LA EDUCACIÓN SUPERIOR ARGENTINA
}

\author{
Inequality Gaps in the Access to Higher Education in Argentina
}

\author{
Cecilia Adrogué ${ }^{1}$ \\ ORCID: 0000-0002-3716-7352 \\ Ana García de Fanelli² \\ ORCID: 0000-0001-7736-2868 \\ ${ }^{1}$ Consejo Nacional de Investigaciones Científicas y Técnicas \\ Universidad de San Andrés, Argentina \\ ${ }^{2}$ Centro de Estudios de Estado y Sociedad \\ Consejo Nacional de Investigaciones Científicas y Técnicas, Argentina \\ Correspondencia: cadrogue@gmail.com
}

Recibido: 07/07/2021

Aceptado: 28/09/2021

Resumen: En un contexto de mecanismos de admisión poco selectivos y gratuidad de los estudios en el sector público, la demanda social por estudios de educación superior creció fuertemente en Argentina. Este artículo analiza si el acceso a la educación superior argentina presenta patrones de desigualdad social. Para ello se estudia si la probabilidad de acceso a la educación superior que tiene cada egresado del nivel secundario difiere según su posición en la estratificación económica, el nivel educativo del hogar y el género. Tras estimar un modelo de regresión logística se encontró que las chances de acceso a la educación superior son menores para los jóvenes de nivel socioeconómico bajo y primera generación con educación universitaria. La juventud que habita hogares de menor ingreso económico y capital cultural tiene además mayor probabilidad de estudiar carreras terciarias que universitarias y de asistir a una institución pública que privada.

Palabras clave: enseñanza superior; acceso a la educación; equidad educativa; desigualdad social; capital cultural.

\begin{abstract}
In a context of limited selective admission mechanisms and tuition free studies in the public sector, Argentina's social demand for higher education has grown vigorously. This article analyzes whether access to higher education in Argentina presents patterns of social inequality. To that end, the article analyzes whether each high school graduate's participation differs according to their position in the economic stratification, the cultural capital of the home and the gender. After estimating a logistic regression model, we found that the chances to access higher education are lower for young people at the low socioeconomic level and those who are a first generation with university education. Youths living in households with lower economic income and cultural capital are also more likely to study tertiary fields than university ones and to attend a public institution than a private one.
\end{abstract}

Keywords: higher education; access to education; educational equity; social inequality; cultural capital. 


\section{Introducción}

Al igual que ha ocurrido en otros países en el plano internacional (Brunner y Miranda, 2016; Brunner y Labraña, 2020; Haimovich Paz, 2017; Marginson, 2016), la demanda social por estudios de educación superior ha crecido vigorosamente en Argentina, especialmente desde mediados de los años ochenta (Buchbinder y Marquina, 2008). En América Latina, se implementaron políticas públicas tendientes a mejorar la participación de los grupos en desventaja social en la escuela secundaria y en el nivel superior. En este último caso, los países incorporaron políticas especiales de admisión y de financiamiento y expandieron la oferta de instituciones y programas con el fin de mejorar las chances de acceso a este nivel de los grupos antes excluidos (Balán, 2020). En Argentina, al igual que en el resto de América Latina, se elevó la escolarización superior de las y los jóvenes de menor ingreso socioeconómico. Así, entre el año 2003 y el 2019, la participación en la educación superior de la juventud de 18 a 20 años perteneciente a los hogares del primer quintil de ingreso per cápita familiar aumentó de 36 a $48 \%{ }^{1}$

Esta fuerte expansión de la matrícula de educación superior permitió que, en el año 2017, Argentina alcanzara una tasa bruta de escolarización superior de $63 \%$ para el grupo etario de 18 a 24 años (García de Fanelli, 2019). Siguiendo la clasificación de Trow (2006), al traspasar el umbral del $50 \%$ en este indicador, Argentina ingresó al grupo de países con educación superior universal o a los de alta participación, según la definición de Marginson (2016).

Si bien el mayor acceso a la educación superior, es decir su democratización, se asocia con una mejora de la equidad en la medida que aumenta la participación en la matrícula de los sectores desaventajados social y económicamente (Raftery y Hout, 1993; Vieira do Nascimento et al., 2020), persisten patrones de desigualdad social. Al respecto Chiroleu (2014) distingue entre un sentido amplio y otro restringido del concepto de democratización aplicado a la educación superior. Según el primero, la democratización es sinónimo de extensión del derecho a la educación superior a un número elevado de personas. El sentido restringido de democratización, también analizado por Ezcurra (2020a), supone que los diferentes grupos sociales que acceden a la educación superior tienen oportunidades equivalentes de obtener resultados similares. Esta segunda acepción, que pone el acento en la desigualdad social, se corresponde con enfoques como

\footnotetext{
${ }^{1}$ Estimación propia en base a información de la Encuesta Permanente de Hogares (EPH) para los años 2003 y 2019 respectivamente.
} 
el de Lucas (2001, 2017), denominado Effectively Maintained Inequality (EMI). ${ }^{2}$ Lucas destaca que las desigualdades de acceso a la educación superior, asociadas con el nivel socioeconómico y cultural de los estudiantes, persisten en procesos de masificación de la educación superior debido a la estratificación jerárquica de las instituciones y carreras según prestigio, calidad y, en ocasiones, costos de enseñanza.

Este debate sobre la masificación y la equidad en la educación superior es especialmente relevante en Argentina. El grado avanzado de matriculación en la educación superior podría hacer suponer que existe igualdad de oportunidades en el acceso. Este artículo tiene por objetivo principal analizar en qué medida el acceso a la educación superior argentina presenta patrones de desigualdad social. Se lo considerará de tal manera en la medida en que el acceso a la educación superior esté asociado con variables adscriptivas tales como el nivel socioeconómico y educativo del hogar y el género (Organisation for Economic Co-operation and Development [OECD], 2018).

En el primer apartado de este artículo se analizan los enfoques teóricos que buscan explicar por qué las elecciones de carreras e instituciones de educación superior por parte de los egresados del secundario difieren según su posición socioeconómica y el nivel educativo del hogar. En el segundo apartado se plantean las hipótesis que guían la investigación y se brinda información descriptiva básica sobre el sistema de educación superior argentino. En el tercer apartado se exponen los datos y el método empleado en esta investigación y se examinan las características de la base de datos utilizada y las metodologías propuestas para el análisis de los datos. En el cuarto apartado se da cuenta de los hallazgos que surgen del análisis descriptivo y de la estimación de los modelos de regresión logística propuestos, para luego concluir señalando cuáles son los principales rasgos que presenta la masificación de la educación superior argentina en lo que respecta a la equidad en el acceso.

\section{Persistencia de la desigualdad social en el acceso a la educación superior}

En la medida en que los jóvenes de los hogares de menor ingreso y capital cultural se incorporan crecientemente a la educación superior, las familias de nivel socioeconómico alto y medio alto despliegan estrategias para asegurar mantener el valor de la credencial educativa que

\footnotetext{
${ }^{2}$ Esta denominación proviene de la crítica de Lucas al enfoque de Maximally Maintained Inequality (MMI) de Raftery y Hout (1993), quienes sostienen que el crecimiento de la matrícula y del número de instituciones de educación superior y de las carreras, favorece que los jóvenes de menores ingresos eleven su participación en la educación superior.
} 
obtienen sus hijos. Por ello, las instituciones de educación superior seleccionadas por los sectores de clase alta y media son aquellas de mayor prestigio y calidad y los programas de grado también de alto estatus (Lucas, 2017; Trow, 2006). En tal caso, la expansión en la escolarización en el nivel superior no garantiza el aumento de la equidad en el acceso, sino que, por el contrario, contribuye a reproducir las desigualdades de ingresos y capital cultural preexistente. Esto da lugar a lo que Ezcurra $(2019,2020 b)$ denomina "democratización estratificada".

En suma, la toma de decisión por parte del estudiante respecto de a qué institución de educación superior concurrir y qué carrera elegir suele estar asociada con el perfil socioeconómico y cultural de su hogar de pertenencia. La discusión sobre este tema se remonta a la distinción que realizó Boudon (1974) respecto a las diferencias en el rendimiento académico según clases sociales. Destaca Boudon que los estudiantes de los sectores socioeconómicamente más altos tienden a obtener mejores resultados en su aprendizaje escolar producto del proceso de socialización que han recibido dentro de hogares con mejores recursos y capital cultural. Como consecuencia, los egresados del nivel secundario, provenientes de estos sectores más acomodados socialmente, optan por continuar estudios universitarios y tienden a elegir carreras de mayor exigencia académica que los jóvenes de sectores socioeconómicamente más bajos. No obstante, Boudon también señaló que el efecto de clase sobre las decisiones que toman los jóvenes y sus familias no ocurre sólo por este motivo. Destaca además la existencia de un efecto secundario, que refuerza la desigualdad social. A igualdad de rendimiento académico en los estudios secundarios, los jóvenes de los sectores más bajos suelen elegir alternativas de estudios que consideran menos exigentes y selectivas. Reforzando estas hipótesis, estudios recientes encuentran que la educación de la madre y el padre (capital cultural del hogar) es uno de los factores más potentes para asegurar la ventaja social a través del sistema escolar previo a la transición a la universidad (Chesters, 2015).

La importancia del capital cultural del hogar como determinante de la elección y el posterior acceso a la educación superior queda expuesta en las investigaciones sobre los así llamados "estudiantes de primera generación", es decir aquellos alumnos cuyas madres o padres no asistieron a la universidad (Choy, 2001). Los estudiantes primera generación tienden a seleccionar como campo de estudio de nivel superior las carreras de corte vocacional o técnico en lugar de las académicas (Chen y Carroll, 2005). Según los hallazgos de distintas investigaciones, los estudiantes primera generación se encuentran en desventaja en lo que atañe al conocimiento sobre costos y procesos de matriculación en la educación superior y también tienen menores expectativas respecto del logro del título universitario (Pascarella et al., 2004). 
La existencia de decisiones o estrategias educativas diferentes en los agentes según clase social también está presente en la teoría de la reproducción de Bourdieu y Passeron (2004). Según estos autores, los sectores dominantes despliegan estrategias para conservar su posición social. Además, existe un conjunto de acciones pedagógicas destinadas a reproducir el habitus que corresponden a los intereses de estas clases, lo cual lleva a que, cuando los jóvenes de las clases privilegiadas acceden a las universidades, se sientan "como peces en el agua" frente al extrañamiento y distanciamiento social que experimentan los jóvenes de las clases bajas.

Dentro del enfoque del individualismo metodológico, se sostiene que la ambición académica de los jóvenes que pertenecen a los hogares de bajo nivel socioeconómico es más limitada, ya que lo que buscan es sostener la posición de clase de sus progenitores. Por el contrario, los sectores de clase alta se vuelcan a los estudios universitarios, dado que solo de este modo pueden mantener su posición social (Breen y Goldthorpe, 1997; Valdés Fernández, 2019). Este mecanismo de aversión relativa al riesgo o "efecto secundario" en Boudon, se considera un factor central en la explicación del factor clase en la transición entre el nivel secundario y la enseñanza superior (Barone et al., 2018). Los miembros de todas las clases comparten la misma aversión al riesgo, el temor a la movilidad descendente. El punto de partida, empero, difiere según el sector de origen. En la investigación empírica, lo que se ha observado es que los estudiantes de nivel socioeconómico bajo, aun cuando hayan obtenido buenas calificaciones en la escuela secundaria, suelen preferir inscribirse en programas educativos e instituciones menos exigentes y riesgosos, que garantizan retornos ocupacionales semejantes a los de sus progenitores (Troiano et al., 2019).

Los hallazgos de investigaciones en el plano internacional dan cuenta de la segmentación según tipo de carrera elegida (académica versus vocacional) dependiendo del capital cultural del hogar. Una encuesta realizada en ocho países (Finlandia, la comunidad flamenca de Bélgica, Francia, Israel, Holanda, Noruega, Suecia y Estados Unidos) reveló que los estudiantes con madres y padres con bajo nivel educativo estaban sustancialmente sobrerrepresentados en los programas vocacionales. De hecho, en casi todos los países o economías con datos disponibles, la proporción de estudiantes cuyos progenitores no habían alcanzado el nivel de educación superior es al menos dos veces mayor entre los participantes en programas vocacionales que entre los asistentes a programas generales (OECD, 2019). De igual modo, un estudio realizado en Brasil, Colombia y México muestra la mayor representación de los estudiantes primera generación y de los estratos de menores ingresos en las instituciones terciarias no universitarias, centralmente focalizadas en la formación vocacional y técnica (García de Fanelli y Jacinto, 2010). 
En suma, la literatura analizada muestra que el acceso a la educación superior es desigual en tanto está asociado con características económicas y culturales de los hogares de las y los jóvenes y por la presencia de circuitos diferenciales de acceso según tipo de instituciones estatales y privadas y de carreras académicas o vocacionales o técnicas. Determinar si efectivamente estos patrones de desigualdad se presentan también en la educación superior argentina es relevante para el diseño de políticas educativas. En particular, la presencia de esta situación de inequidad destaca la importancia de incorporar políticas públicas e institucionales destinadas a brindar información y acompañamiento al alumnado más desaventajado social y económicamente en el tránsito entre la educación secundaria y la superior.

\section{Hipótesis en el marco de la educación superior argentina}

Para el análisis de los factores asociados con el acceso a la educación superior en Argentina se partió de dos hipótesis. Primero, se sostiene que las chances de acceso a la educación superior son menores para aquellos egresados del nivel secundario pertenecientes a hogares de nivel socioeconómico bajo y para los que son primera generación en su familia con educación universitaria. Segundo, se considera que los jóvenes pertenecientes a hogares de menores ingresos y primera generación en el hogar con educación universitaria tienen más probabilidad de estudiar carreras terciarias no universitarias que universitarias y tienen también mayor presencia en el sector público que en el sector privado.

A los fines de aclarar lo expresado en las hipótesis, es importante dar cuenta de algunos rasgos básicos de la configuración del sector de educación superior de Argentina.

En Argentina hay 131 instituciones universitarias, 67 de las cuales son estatales y 64 privadas. En estas instituciones se imparten carreras de pregrado y grado, dentro de las cuales se encuentran las principales carreras profesionales que habilitan al ejercicio profesional. Cabe destacar que entre fines de los años ochenta y el 2020 se crearon 38 instituciones universitarias estatales y 41 privadas, lo cual amplió la accesibilidad a la educación universitaria en distintas regiones del país (García de Fanelli \& Balán, 1994; Argentina Gobierno, 2020a). Además del sector universitario, existe una amplia oferta de instituciones terciarias estatales y privadas (2.279) distribuidas a lo largo de todo el país, que brindan formación docente y técnica (Argentina Gobierno, 2020b). 
Los mecanismos de admisión que se implementan en la mayoría de las instituciones de educación superior de Argentina son en principio no selectivos. Los aspirantes no deben aprobar exámenes generales al final de la escuela media o al momento de su inscripción en las instituciones de educación superior y tampoco se suele establecer un número máximo de aspirantes a ingresar por programa. En el sector universitario se observa, no obstante que los tipos y modalidades de acceso muestran la presencia de múltiples sistemas de admisión (Fernández Lamarra et al., 2018; Marquina, 2011). La modalidad más frecuente es a través de cursos o exámenes preuniversitarios que los aspirantes deben aprobar como condición para ser considerados alumnos en las carreras de grado. En estos casos, no es claro en qué medida estos cursos tienen un propósito nivelatorio del conocimiento de los estudiantes o selectivo (Cambours y Gorostiaga, 2019). En unos pocos casos, especialmente en algunas carreras de grado de medicina, el ingreso es mediante exámenes y cupos. Por el contrario, el acceso a las carreras terciarias no supone la realización de cursos o exámenes previos, con la excepción de algunas carreras artísticas o de la enseñanza de lenguas extranjeras (Kisilevsky, 2002). Cabe además tener presente que no se cobran aranceles para el estudio de las carreras de grado o pregrado, tanto en universidades como en los institutos superiores no universitarios estatales. Por el contrario, el sector privado cobra aranceles, el cual varía según la carrera y el prestigio de las instituciones (Rabossi, 2011).

Como se observa en la Figura 1, más de la mitad de la matrícula de pregrado y grado se concentra en el sector universitario estatal. Le sigue en importancia el sector terciario estatal. Finalmente, en el sector privado universitario y terciario estudia aproximadamente un cuarto del alumnado de pregrado y grado. 


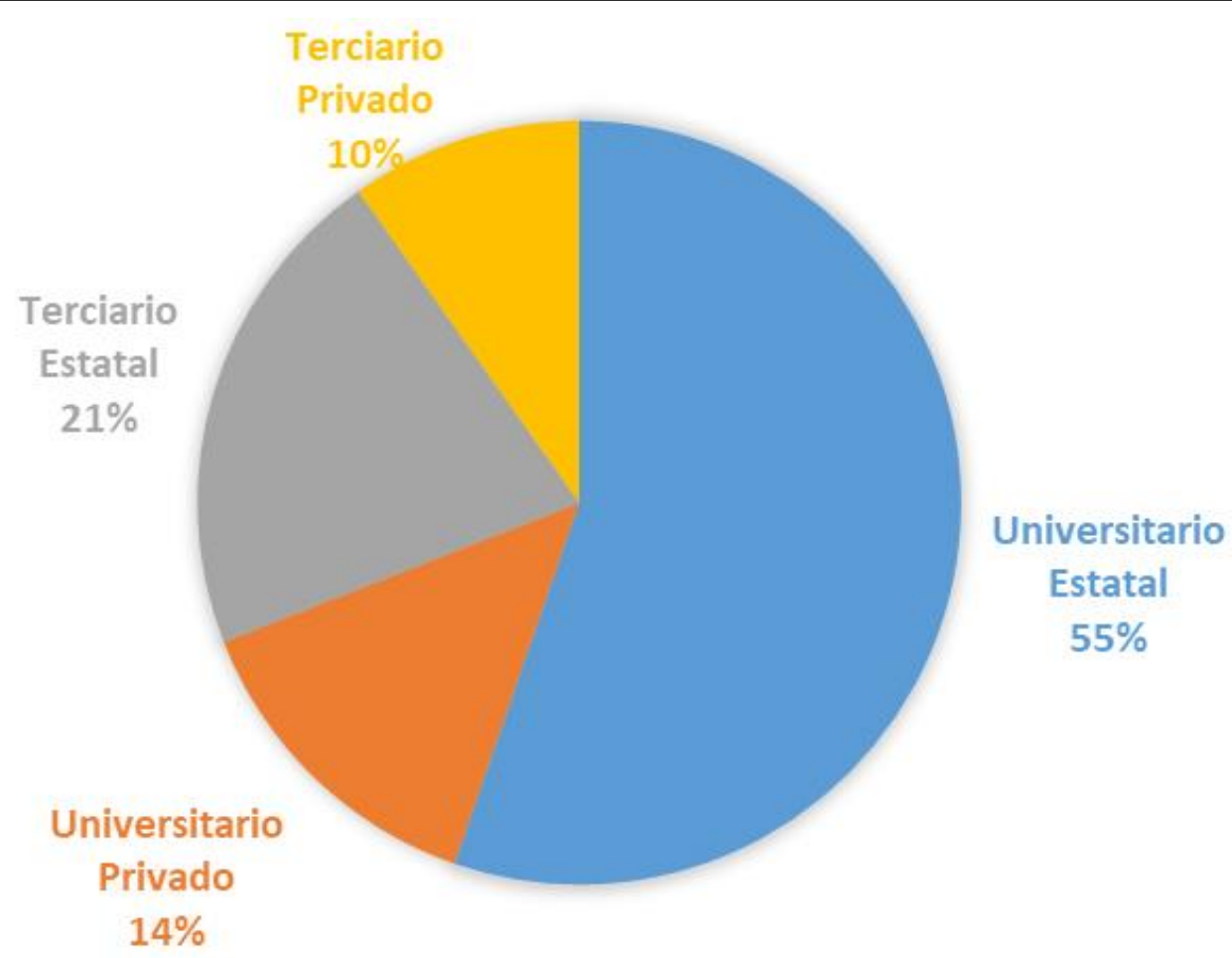

Figura 1. Distribución de la matrícula de pregrado y grado en la educación superior argentina según tipo de institución y gestión, 2020. Fuente: elaboración propia sobre la base de Argentina Gobierno (2020a, 2020b)

Por último, una dimensión que permite demarcar la estratificación que existe entre las carreras universitarias y las terciarias no universitarias en Argentina es su efecto sobre el ingreso en el mercado de trabajo. Los egresados de carreras universitarias y de carreras terciarias obtienen retornos privados diferentes en el mercado laboral argentino, siendo en promedio $50 \%$ superiores en el primer caso. En 2017, los salarios horarios de los individuos con universitario completo y con terciario no universitario completo respecto de los trabajadores con primario incompleto eran $75 \%$ y $50 \%$ superiores respectivamente (Levy Yeyati et al., 2018). Además, un estudio cualitativo sobre la percepción de los estudiantes de educación superior en Argentina muestra que los jóvenes consideran a la educación universitaria como la mejor opción frente a la terciaria no universitaria. $\mathrm{Al}$ respecto, los jóvenes destacan que los estudios universitarios brindan mayores chances de éxito dado que gozan de mayor prestigio entre los empleadores. Recalcan asimismo que la formación en las carreras de educación terciaria no universitaria es más especializada, limitando la posibilidad de progresión profesional y reconversión laboral (Veleda, 2002).

En suma, la segunda hipótesis se fundamenta en la presencia de dos tipos de carreras, universitarias y terciarias no universitarias, que se diferencian por los títulos que brindan, siendo 
los títulos académicos y profesionales de grado reservados a las instituciones universitarias. Asimismo, según las investigaciones antes señaladas, las carreras universitarias son percibidas como más prestigiosas que las terciarias, ofreciendo mayor flexibilidad en la inserción laboral y un mayor retorno económico en el mercado de trabajo.

Otra dimensión de estratificación se produce por el costo de las carreras, gratuito en el sector público y con aranceles en el sector privado. Dado que las principales becas disponibles en el nivel superior para los estudiantes con mayor vulnerabilidad económica no llegan a cubrir el costo de oportunidad de estudiar sin tener que trabajar al mismo tiempo, el cobro de aranceles en el sector privado constituye una barrera institucional importante para el acceso de los sectores de nivel socioeconómico más bajo (García de Fanelli, en prensa).

\section{Datos y método}

Se construyó una base de datos original compuesta por un pool de datos ${ }^{3}$ que contiene todas las ondas disponibles de la Encuesta Permanente de Hogares (EPH) entre los años 2003 y 2019, de forma tal de contar con información referida a los jóvenes argentinos que acceden a la educación superior. Se utilizó este período por ser el más amplio disponible con información comparable para Argentina. La EPH es una encuesta nacional representativa de la población urbana del país, que desde el año 2003 es continua con periodicidad trimestral. La encuesta incluye preguntas relativas a las características demográficas, educativas, laborales y socioeconómicas de la población.

En primer lugar, se realiza un análisis descriptivo de los datos y luego se estiman tres modelos de regresión.

Para estudiar cuál es el grado de relación entre los factores socioeconómicos, demográficos y la educación de la madre y el padre y la probabilidad de participación de los jóvenes en el nivel superior, siguiendo a Cabrera (1994), se emplea un modelo de regresión logística. ${ }^{4}$ Se estiman los parámetros del siguiente modelo:

\footnotetext{
${ }^{3}$ Se denomina pool de datos ya que corresponde a un agrupamiento de datos de corte transversal o cross section en grupos más abarcativos (Ahumada et al., 2018), en el caso en cuestión son datos correspondientes a trimestres y años consecutivos.

${ }^{4}$ Para análisis similares en la Argentina, han utilizado esta técnica entre otros Gasparini (2002) y Paz y Cid (2012).
} 
$\operatorname{Pr} o b($ estudiar $=1)=F(X \beta)$

Aquí, la variable a explicar es la probabilidad de continuación de estudios de nivel superior entre las personas egresadas de la escuela secundaria. Esta es una variable dicotómica, que tiene valor igual a 1 si la persona realiza estudios superiores y 0 en caso contrario. Cabe al respecto señalar que más del $60 \%$ de los jóvenes de 18 a 20 años que han concluido la escuela secundaria declara realizar estudios superiores. $\beta$ es el vector de coeficientes que se quiere estimar y $X$ representa aquellas variables observables correspondientes a las características de la persona, de su familia y del hogar en el que vive, que inciden en la probabilidad de realizar estudios superiores.

En este estudio econométrico, la muestra está compuesta por los jóvenes de 18 a 20 años que realizan estudios superiores y que habitan en su hogar en calidad de hijos. Esta selección responde al objetivo de poder contar con la información de la educación de sus padres y del nivel socioeconómico de su familia de origen. En esta situación se encuentra el $83 \%$ de los estudiantes de nivel superior de 18 a 20 años. Se han incluido, además de aquellos estudiantes que son hijos del jefe de hogar (92\% de la muestra), a los que son yernos/nueras (2\%) y nietas/os (6\%). Se entiende que la emancipación del hogar de origen, con todas las consecuencias que acarrea en cuanto al ámbito laboral, maternal/paternal, posiblemente se relacione con la decisión de continuar o no estudios superiores en un contexto de expansión de la matrícula estudiantil. No obstante, esta situación es menos probable en el grupo etario seleccionado en este análisis (18 a 20 años), siendo más frecuente entre los estudiantes "no tradicionales” (Gilardi y Guglielmetti, 2011).

Las variables explicativas $(X)$ consideradas son las siguientes:

- Género: Indica el sexo del individuo, 1 si es varón, 0 si es mujer. El 45 \% de la muestra está compuesta por varones y el $55 \%$ por mujeres.

- Edad: Es una variable que asume valor 18, 19 o 20 según los años del individuo. La edad promedio de la muestra analizada es de 19,11 años.

- Primera generación: Tiene valor igual a 1 si ni el jefe de hogar ni su cónyuge han realizado estudios universitarios, y 0 en caso contrario. El $79 \%$ de los jóvenes que ha terminado la educación secundaria es primera generación universitaria.

- Clase media: Asume valor 1 si el nivel de ingreso per cápita familiar pertenece al segundo, tercer o cuarto quintil, 0 en caso contrario. El 57,3 \% de los jóvenes de la muestra pertenece a la clase media. 
- Clase alta: Asume valor 1 si el nivel de ingreso per cápita del hogar pertenece al quinto quintil y 0 en caso contrario. El 8,4 \% de los jóvenes pertenece a la clase alta. La variable omitida es clase baja, situación en la que se encuentran el 34,3\% de los jóvenes que concluyeron la educación secundaria.

Adicionalmente, para estudiar los factores asociados con la elección del tipo de estudios superiores se realizó una estimación de un modelo de regresión logística, pero en este caso el objetivo es evaluar cuánto inciden los factores descriptos en la probabilidad de estudiar carreras terciarias versus universitarias. Para ello se estiman los parámetros del siguiente modelo:

$\operatorname{Pr} o b($ terciario $=1)=F(X \beta)$

La variable explicada es una variable dicotómica que adquiere valor 1 si el estudiante realiza estudios superiores no universitarios y 0 si realiza estudios universitarios. El objetivo es analizar cuán relacionadas están las variables explicativas $X$ descriptas anteriormente con la probabilidad de realizar estudios de nivel terciario no universitario $(\beta)$.

Por último, se estima la probabilidad de que los estudiantes asistan a una institución pública en lugar de una privada. Para ello se estiman los parámetros del siguiente modelo de regresión logística:

$\operatorname{Pr} o b($ pública $=1)=F(X \beta)$

En este caso la variable independiente tiene valor 1 si el joven realiza sus estudios superiores en una institución de gestión pública y 0 si es de gestión privada. $\beta$ es el vector de coeficientes que se quiere estimar y representa cuán relacionadas están las variables observables $X$ con la probabilidad de asistir a una institución pública. Cabe mencionar que, en este caso como en otros estudios de cuestiones educativas, se presenta el grado de relación entre las variables, sin hablar de causalidad. Como prueba de robustez — siguiendo a Angrist y Pischke (2008), quienes afirmaron que las estimaciones de los modelos de probabilidad lineales son similares a los modelos generalizados, con la ventaja de que los coeficientes tienen una interpretación directa-, se ha estimado también el modelo de mínimos cuadrados ordinarios para cada uno de los tres modelos logísticos. 


\section{Resultados y discusión}

A fin de realizar el análisis descriptivo de los datos, se agrupan los resultados según clase social a partir de la posición de los hogares en la distribución del ingreso per cápita familiar. Para ello, siguiendo a Groisman (2016), se considera que integran la clase baja los individuos que pertenecen a los hogares del primer quintil de ingresos; la clase media, aquellos que se ubican en los hogares de los quintiles segundo, tercero y cuarto, y la clase alta, los que se sitúan en los hogares del quinto quintil de ingreso per cápita familiar.

Tal como puede apreciarse en la Figura 2, el 54 \% de los jóvenes entre los 18 y 20 años ha concluido los estudios de nivel secundario, siendo superior la proporción de mujeres que logran completar este nivel.

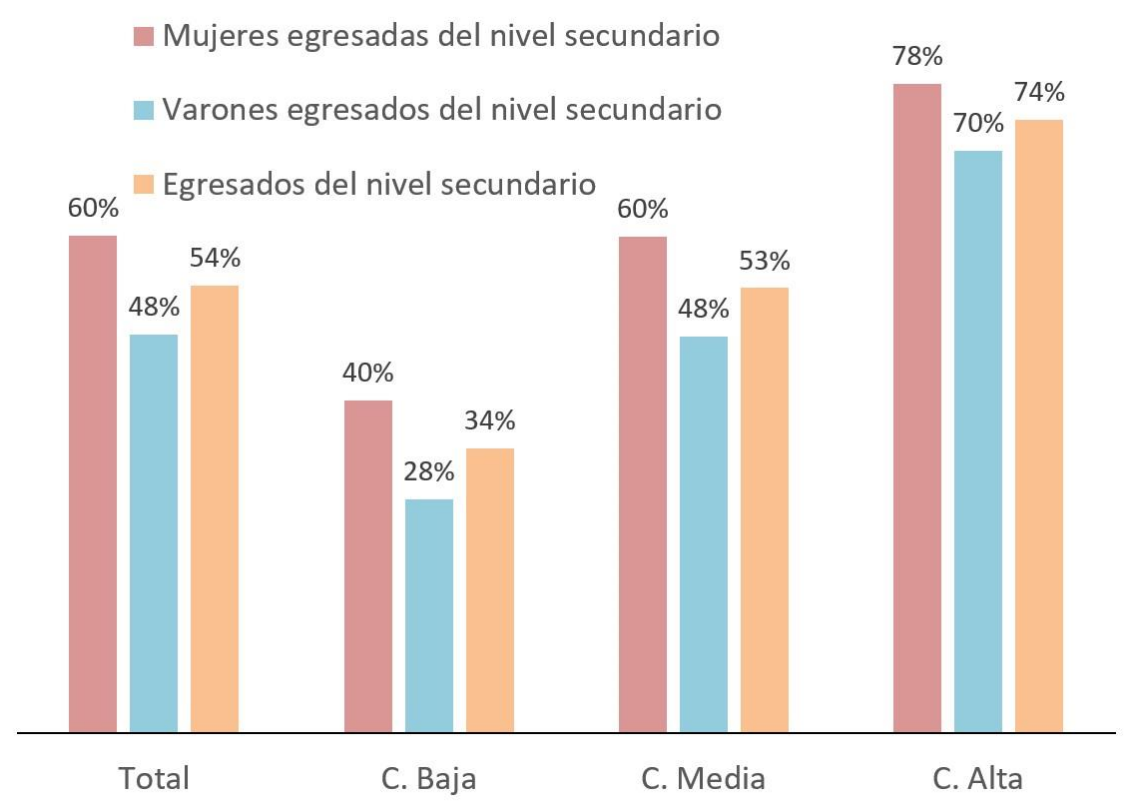

Figura 2. Proporción de jóvenes de 18 a 20 años egresados del nivel secundario total y según género y clase social. Fuente: Elaboración propia en base a la EPH 2003-2019.

La brecha en la tasa de completitud en el nivel secundario entre la clase baja y alta también es pronunciada: el doble entre las mujeres y 1,4 veces entre los varones. 
Haber concluido los estudios secundarios constituye una condición necesaria para acceder al nivel superior. ${ }^{5}$ De estos jóvenes de 18 a 20 años que concluyen el nivel secundario, el $65 \%$ de las mujeres y el $57 \%$ de los varones continúan estudiando en el nivel superior. Nuevamente, aquí se observa una gran disparidad según clase social. Menos de la mitad de los que terminan el nivel secundario y tienen bajos ingresos realizan estudios superiores, mientras qué más de ocho de cada diez de aquellos de mayores ingresos familiares siguen estudiando. Entre las mujeres, la brecha según clase es más pronunciada que entre los varones (Figura 3).

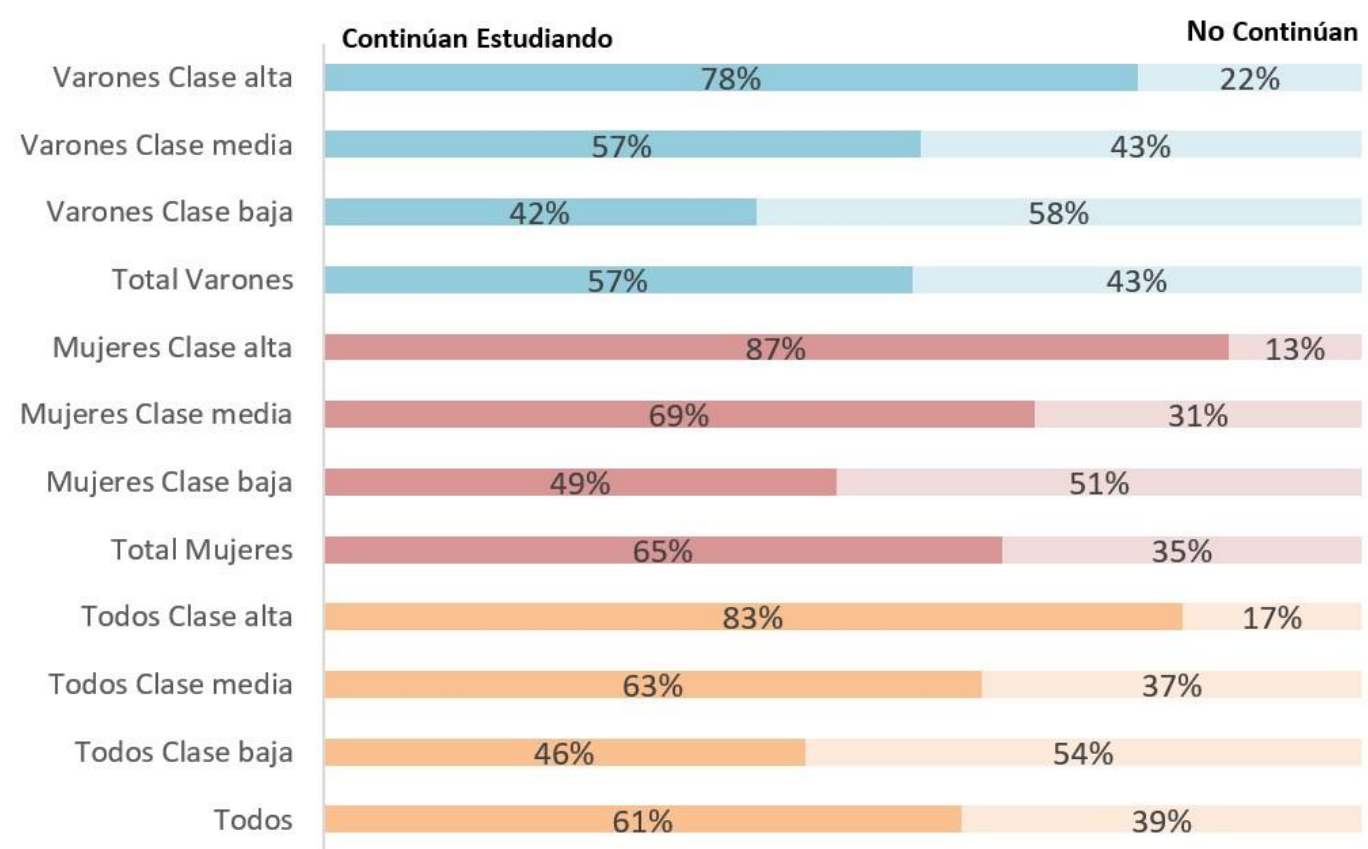

Figura 3. Proporción de jóvenes de 18 a 20 años egresados del nivel secundario, según continuación o no de los estudios superiores, género y clase social. Fuente: Elaboración propia en base a la EPH 2003-2019.

En suma, mientras que poco más de la mitad de los jóvenes de 18 a 20 años concluye el nivel secundario, el $61 \%$ de estos egresados continúa estudiando en el nivel superior. Tanto entre los que finalizan el nivel secundario, como en los que acceden la educación superior, se observan importantes brechas según nivel socioeconómico del hogar y el género.

\footnotetext{
${ }^{5}$ Según la Ley de Educación Superior Argentina, sancionada en 1995, solo los jóvenes de 25 años y más sin título secundario pueden acceder a la educación superior tras rendir un examen de conocimientos en la institución de educación superior.
} 
A continuación, a fin de analizar la relación entre las variables explicativas y sobre la probabilidad de acceder a la educación superior manteniendo las demás características constantes, se presentan los resultados obtenidos para cada uno de los tres modelos de regresión descriptos en el apartado de datos y metodología.

Tabla 1

Resultados de las estimaciones de los modelos MCO y Logit de la probabilidad de realizar estudios superiores

\begin{tabular}{|c|c|c|c|c|c|c|}
\hline & $\begin{array}{c}\text { Coeficiente } \\
\text { MCO }\end{array}$ & & $\begin{array}{l}\text { Coeficiente } \\
\text { Logit }\end{array}$ & & $\begin{array}{c}\text { Razón de } \\
\text { probabilidad Logit }\end{array}$ & \\
\hline \multirow[t]{2}{*}{ Género } & -0.120 & $* * *$ & -0.572 & $* * *$ & 0.564 & $* * *$ \\
\hline & $(0.0032)$ & & 0.016 & & 0.009 & \\
\hline \multirow[t]{2}{*}{ Edad } & -0.009 & $* * *$ & -0.043 & $* * *$ & 0.958 & $* * *$ \\
\hline & $(0.0020)$ & & 0.010 & & 0.009 & \\
\hline \multirow[t]{2}{*}{ Primera Generación } & -0.267 & $* * *$ & -1.491 & $* * *$ & 0.225 & $* * *$ \\
\hline & $(0.0042)$ & & 0.025 & & 0.006 & \\
\hline \multirow[t]{2}{*}{ Clase media } & 0.124 & $* * *$ & 0.558 & $* * *$ & 1.747 & $* * *$ \\
\hline & $(0.0036)$ & & 0.017 & & 0.029 & \\
\hline \multirow[t]{2}{*}{ Clase alta } & 0.200 & $* * *$ & 1.035 & $* * *$ & 2.816 & $* * *$ \\
\hline & $(0.0067)$ & & 0.037 & & 0.103 & \\
\hline \multirow[t]{2}{*}{ Constante } & 0.866 & $* * *$ & 1.975 & $* * *$ & 7.206 & $* * *$ \\
\hline & $(0.0403)$ & & 0.194 & & 1.395 & \\
\hline Dummies por año & Sí & & Sí & & Sí & \\
\hline Dummies por trimestre & Sí & & Sí & & Sí & \\
\hline Dummies por región & Sí & & Sí & & Sí & \\
\hline Observaciones & 80,986 & & 80,986 & & 80,986 & \\
\hline (Pseudo) R2 & 0.109 & & 0.092 & & 0.092 & \\
\hline
\end{tabular}

Fuente: Elaboración propia en base a la EPH 2003-2019.

***Significativo al $99 \%$.

Tal como puede observarse en la Tabla 1, 87 de cada 100 jóvenes que termina la escuela media en Argentina y vive con sus progenitores (o suegros o abuelos), realiza estudios superiores (ver constante de $\mathrm{MCO}$ ). A igualdad de las demás características, los varones tienen menor probabilidad de realizar estudios superiores que las mujeres — por cada diez mujeres, se espera que participen de la educación superior seis varones (ver columna de razón de probabilidad). Haimovich Paz (2017) analiza un conjunto de países latinoamericanos y encuentra un resultado similar, tanto condicionado - es decir, aislando los efectos de las otras características - como no condicionado. En ambos casos, encuentra que las mujeres presentan una participación en educación superior aproximadamente 10 puntos porcentuales superior que los varones. 
Se comprueba también que aquellos cuyas madres o padres no tienen educación universitaria poseen una probabilidad considerablemente menor de realizar estudios superiores. Se espera que por cada 2 que se anotan de primera generación, lo hagan 10 jóvenes cuyas madres o padres tienen estudios universitarios. Este resultado está en línea con la literatura que analiza los logros de los estudiantes primera generación con educación superior (Choy, 2001) y con la sociología de la desigualdad social en la participación educativa según clase (Boudon, 1974; Bourdieu y Passeron, 2004). En América Latina, Haimovich Paz (2017) halló que aquellos jóvenes cuyos padres han terminado la educación media tienen en promedio 38 puntos porcentuales más de probabilidad de acceder a la educación superior.

Por último, lo que se desprende del análisis de la estadística descriptiva expuesta en las Figuras 2 y 3 , sin aislar los demás efectos, se verifica nuevamente aquí, aislando todos los demás efectos, tales como aquellos relacionados con el género, la edad y la educación de la madre y el padre. Por cada 100 personas de clase baja que estudian en el nivel superior, se encuentran 175 de clase media y 281 de clase alta. Cabe mencionar que para realizar el análisis se ha controlado por la región en la que habita el joven o la joven en cuestión, dado que la oferta no es igual en todas las regiones del país, lo cual afecta sin duda, la probabilidad de que los jóvenes puedan enrolarse en estudios superiores.

En la Tabla 2 se analizan los factores que inciden en la probabilidad de realizar estudios terciarios no universitarios versus universitarios. 
Tabla 2

Resultados de las estimaciones de los modelos MCO y Logit de la probabilidad de realizar estudios terciarios vs. estudios universitarios

\begin{tabular}{|c|c|c|c|c|c|c|}
\hline \multirow[b]{2}{*}{ Género } & \multicolumn{2}{|l|}{$\begin{array}{c}\text { Coeficiente } \\
\mathrm{MCO}\end{array}$} & \multirow{2}{*}{$\begin{array}{c}\text { Coeficiente } \\
\text { Logit } \\
-0.234\end{array}$} & \multicolumn{3}{|c|}{$\begin{array}{c}\text { Razón de } \\
\text { probabilidad Logit }\end{array}$} \\
\hline & -0.040 & $* * *$ & & $* * *$ & 0.792 & $* * *$ \\
\hline & 0.004 & & 0.022 & & 0.018 & \\
\hline \multirow[t]{2}{*}{ Edad } & 0.039 & $* * *$ & 0.227 & $* * *$ & 1.255 & $* * *$ \\
\hline & 0.002 & & 0.014 & & 0.017 & \\
\hline \multirow[t]{2}{*}{ Primera Generación } & 0.166 & $* * *$ & 1.112 & $* * *$ & 3.041 & $* * *$ \\
\hline & 0.004 & & 0.030 & & 0.091 & \\
\hline \multirow[t]{2}{*}{ Clase media } & -0.034 & $* * *$ & -0.172 & $* * *$ & 0.842 & $* * *$ \\
\hline & 0.004 & & 0.024 & & 0.020 & \\
\hline \multirow[t]{2}{*}{ Clase alta } & -0.056 & $* * *$ & -0.368 & $* * *$ & 0.692 & $* * *$ \\
\hline & 0.007 & & 0.046 & & 0.032 & \\
\hline \multirow[t]{2}{*}{ Constante } & -0.671 & $* * *$ & -6.665 & $* * *$ & 0.001 & $* * *$ \\
\hline & 0.047 & & 0.276 & & 0.000 & \\
\hline Dummies por año & Sí & & Sí & & Sí & \\
\hline Dummies por trimestre & Sí & & Sí & & Sí & \\
\hline Dummies por región & Sí & & Sí & & Sí & \\
\hline Observaciones & 50,423 & & 50,423 & & 50,423 & \\
\hline (Pseudo) R2 & 0.063 & & 0.063 & & 0.063 & \\
\hline
\end{tabular}

Fuente: Elaboración propia en base a EPH 2003-2019.

***Significativo al $99 \%$.

Como puede observarse, las mujeres tienen mayor probabilidad de realizar estudios terciarios no universitarios. Este resultado se relaciona con la importante concentración de mujeres en las carreras de docencia en los niveles preescolar, primario y secundario. Por cada diez mujeres que realizan estudios terciarios no universitarios, lo hacen menos de ocho varones. Además, la probabilidad de realizar estudios terciarios es mayor entre los jóvenes de mayor edad. Es probable que este hallazgo responda a que, según muestran Trombetta (2000) y Kisilevsky (2002), una proporción significativa de los jóvenes que ingresan a los institutos terciarios no universitarios lo hacen después de tener una experiencia no exitosa en los estudios universitarios. Según Kisilevsky (2002), la elección de la educación terciaria no universitaria en Argentina como una "segunda opción" estaría reflejando implícitamente un juicio relativo sobre el prestigio de la universidad frente a las instituciones terciarias. Resultados semejantes también se aprecian en estudios longitudinales que destacan que los jóvenes de menores ingresos suelen tener trayectorias no lineales o estables, siendo común la transferencia del sector universitario al terciario no universitario (Hass y Hadjar, 2020). 
Los estudiantes primera generación también tienen mayor probabilidad de realizar estudios terciarios no universitarios. En contraste, los estudiantes de clase media y alta tienen mayor probabilidad de realizar estudios universitarios en lugar de estudios terciarios no universitarios. Estos hallazgos están en línea con la literatura que estudia el proceso de desigualdad social por efecto de la estratificación del acceso a la educación superior (Lucas, 2017; Trow, 2006; Troiano et al., 2019). En particular, este resultado se corresponde con el estudio realizado por la OECD (2019) que encuentra que los estudiantes de nivel superior cuyos progenitores tienen bajo nivel educativo están sobrerrepresentados en los programas vocacionales.

En la Tabla 3 se presenta la estimación de la ecuación (3). Allí se puede verificar que los estudiantes primera generación y los de clase baja, a igualdad de los demás factores, presentan una mayor probabilidad de estudiar en una institución pública. Por el contrario, aquellos que habitan en hogares con mayor nivel socioeconómico y capital cultural, tienen mayor probabilidad de estudiar en una institución privada, controlando por el resto de las variables. Cabe tener en cuenta al respecto que en Argentina las universidades e institutos terciarios no universitarios estatales no cobran arancel por los estudios de pregrado y grado. 
Tabla 3

Resultados de las estimaciones de los modelos MCO y Logit de la probabilidad de realizar estudios superiores en instituciones públicas vs. privadas.

\begin{tabular}{|c|c|c|c|c|c|c|}
\hline & $\begin{array}{c}\text { Coeficiente } \\
\mathrm{MCO} \\
\end{array}$ & & $\begin{array}{c}\text { Coeficiente } \\
\text { Logit } \\
\end{array}$ & & $\begin{array}{c}\text { Razón de } \\
\text { probabilidad Logit }\end{array}$ & \\
\hline \multirow[t]{2}{*}{ Estudios terciarios } & -0.185 & $* * *$ & -1.145 & $* * *$ & 0.318 & $* * *$ \\
\hline & 0.004 & & 0.026 & & 0.008 & \\
\hline \multirow[t]{2}{*}{ Género } & 0.016 & $* * *$ & 0.108 & $* * *$ & 1.114 & $* * *$ \\
\hline & 0.003 & & 0.024 & & 0.027 & \\
\hline \multirow[t]{2}{*}{ Edad } & -0.004 & $*$ & -0.027 & $*$ & 0.973 & $*$ \\
\hline & 0.002 & & 0.015 & & 0.014 & \\
\hline \multirow[t]{2}{*}{ Primera Generación } & 0.050 & $* * *$ & 0.345 & $* * *$ & 1.412 & $* * *$ \\
\hline & 0.004 & & 0.027 & & 0.038 & \\
\hline \multirow[t]{2}{*}{ Clase media } & -0.063 & $* * *$ & -0.465 & $* * *$ & 0.628 & $* * *$ \\
\hline & 0.004 & & 0.029 & & 0.018 & \\
\hline \multirow[t]{2}{*}{ Clase alta } & -0.142 & $* * *$ & -0.935 & $* * *$ & 0.392 & $* * *$ \\
\hline & 0.007 & & 0.044 & & 0.017 & \\
\hline \multirow[t]{2}{*}{ Constante } & 0.969 & $* * *$ & 2.620 & $* * *$ & 13.736 & $* * *$ \\
\hline & 0.043 & & 0.295 & & 4.054 & \\
\hline Dummies por año & Sí & & Sí & & Sí & \\
\hline Dummies por trimestre & Sí & & Sí & & Sí & \\
\hline Dummies por región & Sí & & Sí & & Sí & \\
\hline Observaciones & 50,396 & & 50,396 & & 50,396 & \\
\hline (Pseudo) R2 & 0.067 & & 0.068 & & 0.068 & \\
\hline
\end{tabular}

Fuente: Elaboración propia en base a EPH 2003-2019 — todas las ondas disponibles. ***Significativo al $99 \%$, **al $95 \%$ y *al $90 \%$.

En suma, tal como se planteó en las hipótesis guías de esta investigación, por un lado, las chances de acceso a la educación superior son menores para aquellos egresados del nivel secundario que pertenecen a hogares de nivel socioeconómico bajo y para los que son primera generación en su familia con educación universitaria. Por el otro lado, los jóvenes pertenecientes a hogares de menores ingresos y primera generación en el hogar con educación universitaria tienen mayor probabilidad de estudiar carreras terciarias no universitarias que universitarias y tienen también mayor probabilidad de asistir a una institución pública que privada.

Los resultados de las estimaciones logísticas permitieron aislar los efectos y confirmar lo que la estadística descriptiva mostraba. Por último, cabe mencionar que los resultados de las estimaciones por Mínimos Cuadrados Ordinarios confirmaron los hallazgos obtenidos a través de las regresiones logísticas; tanto los coeficientes muestran una influencia similar para cada factor presentado, así como la misma significación estadística. 


\section{Conclusiones}

En el contexto de América Latina, Argentina es uno de los países con mayor tasa de matriculación en la educación superior. Acompañando este proceso de aumento de la escolarización superior, la participación de los jóvenes de menores ingresos ha crecido 12 puntos porcentuales entre el 2003 y los 2019. Si bien este logro supone en sí mismo una mejora en la equidad educativa al democratizar el acceso al conocimiento avanzado, en este trabajo se ha establecido como objetivo analizar en qué medida el acceso a la educación superior argentina presenta patrones de desigualdad social. Esto último puede corroborarse al analizar si la participación que alcanza cada joven difiere según su posición en la estratificación económica, el capital cultural de su hogar medido por la educación de la madre y el padre y el género.

Como guía en la investigación realizada se partió de dos hipótesis. Primero, se sostiene que las chances de acceso a la educación superior son menores para aquellos egresados del nivel secundario pertenecientes a hogares de nivel socioeconómico bajo y para los que son primera generación en su familia con educación universitaria. Segundo, se considera que los jóvenes pertenecientes a hogares de menores ingresos y primera generación en el hogar con educación universitaria tienen más probabilidad de estudiar carreras terciarias no universitarias que universitarias y tienen también mayor presencia en el sector público que en el sector privado.

Partiendo de la información disponible en los microdatos de la Encuesta Permanente de Hogares, este estudio permite corroborar la primera hipótesis en la medida en que la probabilidad de acceso de los jóvenes pertenecientes a la clase alta es casi tres veces más elevada que en la clase baja. Esta brecha de equidad se observa también cuando se analiza la educación de los progenitores. Los estudiantes que son primera generación tienen cuatro veces menos chances que aquellos cuyos padres tienen educación universitaria de estudiar en este nivel. Además, la probabilidad de acceso de las mujeres a la educación superior es 1,8 veces la de los varones en sintonía con lo encontrado por Haimovich Paz (2017) para un conjunto de países latinoamericanos. Estos hallazgos, que resultan de estimar el modelo de regresión logística, confirman la información que surge de la estadística descriptiva.

Además de estas brechas que afectan la equidad en la educación superior argentina, la desigualdad social se refuerza al observar que, tal como lo señalado en la segunda hipótesis, las chances de acceder a instituciones universitarias son más altas entre los egresados del nivel secundario pertenecientes a las clases alta y media. Por el contrario, entre los jóvenes provenientes 
de hogares de clase baja y que son primera generación, la probabilidad de estudiar en instituciones terciarias no universitarias es mayor. Estos hallazgos están alineados, por un lado, con los resultados de Trombetta (2000) y Kisilevsky (2002), quienes señalan que los estudios terciarios suelen ser una segunda opción para aquellos que fracasan en la primera opción - estudios universitarios-. Por otro lado, también se corresponden con otros estudios que encuentran asociación entre los estudiantes primera generación y la elección de carreras (Lucas, 2017; Trow, 2006; Troiano et al., 2019). Cabe al respecto tener presente que los graduados de carreras del terciario no universitario en la Argentina obtienen una tasa de retorno en el mercado de trabajo por debajo de la que alcanzan los egresados universitarios.

Finalmente, como es de esperar por los mayores costos de enseñanza, las chances de acceder al sector privado son más elevadas entre las clases alta y media y entre aquellos que habitan hogares con mayor capital cultural.

Una limitación de los hallazgos encontrados reside en la base de datos utilizada. Un análisis adecuado de los factores que afectan la decisión de matriculación debería realizarse idealmente utilizando una encuesta longitudinal, que esté especialmente diseñada para estudiar temas educativos (Cabrera y La Nasa, 2000). Las encuestas longitudinales permiten, por un lado, incorporar variables no disponibles en la Encuesta Permanente de Hogares, tales como la incidencia de los trayectos educativos y laborales previos, cambios en las variables demográficas del hogar (estado civil, hijos, residencia, etc.) y los factores que inciden sobre las decisiones que toman los estudiantes al momento de optar por continuar estudiando en el nivel superior. Lamentablemente, este tipo de encuestas no están disponibles en la Argentina.

En síntesis, el caso argentino revela que, aun con niveles altos de participación en la educación superior, persisten brechas importantes entre los egresados de nivel secundario en lo que respecta a su probabilidad de acceso a la educación superior en general, y a instituciones universitarias o terciarias, según nivel socioeconómico, educación de los progenitores y género. De estos resultados se desprende que políticas que refuercen el conocimiento sobre las opciones de carreras disponibles en las instituciones de nivel superior y orienten a las y los estudiantes en su vocación académica y su futura inserción laboral son muy necesarias para mejorar la equidad en el acceso en la educación superior argentina. 


\section{Referencias}

Ahumada, H. A., Gabrielli, M. F., Herrera Gomez, M. H., \& Sosa Escudero, W. (2018). Una nueva econometría: Automatización, big data, econometría espacial y estructural. Editorial de la Universidad Nacional del Sur.

Angrist, J. D., \& Pischke, J. S. (2008). Mostly harmless econometrics. Princeton University Press.

Argentina Gobierno. (2020a). Síntesis de Información Estadísticas Universitarias $2018-2019$. https://www.argentina.gob.ar/sites/default/files/sintesis_2018-2019_sistema_universitario_argentino_0.pdf

Argentina Gobierno. (2020b). Anuario Estadístico Educativo 2018. Buenos Aires: Ministerio de Educación. https://www.argentina.gob.ar/sites/default/files/sintesis_2018-2019_sistema_universitario_argentino_0.pdf

Balán, J. (2020). Expanding Access and Improving Equity in Higher Education: The National Systems Perspective. En S. Schwartzman (Ed.), Higher Education in Latin America and the Challenges of the $21^{\text {st }}$ Century (pp. 59-75). Springer Nature. https://doi.org/10.1007/978-3-030-44263-7_3

Barone, C., Triventi, M., \& Assirelli, G. (2018). Explaining Social Inequalities in Access to University: A Test of Rational Choice Mechanisms in Italy. European Sociological Review, 34(5), 554-569.

Boudon, R. (1974). Education, Opportunity, and Social Inequality: Changing Prospects in Western Society. WileyInterscience.

Bourdieu, P., \& Passeron, J. C. (2004). Los herederos. Los estudiantes y la cultura. Siglo XXI.

Buchbinder, P., \& Marquina, M. (2008). Masividad, heterogeneidad y fragmentación: el sistema universitario argentino 1983-2007. Universidad Nacional de General Sarmiento.

Breen, R., \& Goldthorpe, J. H. (1997). Explaining Education Differentials: Towards a Formal Rational Action Theory. Rationality and Society, 9(3), 275-305. https://doi.org/10.1177/104346397009003002

Brunner, J. J., \& Labraña, J. (2020). The Transformation of Higher Education in Latin America: From Elite Access to Massification and Universalisation. En S. Schwartzman (Ed.), Higher Education in Latin America and the Challenges of the $21^{\text {st }}$ Century (pp. 31-41). Springer Nature. https://doi.org/10.1007/978-3-030-44263-7_3

Brunner, J. J., \& Miranda, D. A. (Eds.) (2016). Educación Superior en Iberoamérica. Informe 2016. UNIVERSIACINDA. https://cinda.cl/publicacion/educacion-superior-en-iberoamerica-informe-2016/

Cabrera, A. F. (1994). Logistic regression analysis in higher education: An applied perspective. En J. C. Smart (Ed.), Higher education: Handbook of theory and research Vol. 10 (pp. 225-256). Agathon Press.

Cabrera, A. F., \& La Nasa, S. M. (2000). Three Critical Tasks America's Disadvantaged Face on Their Path to College, New Directions for Institutional Research, (107), 23-29.

Cambours, A., \& Gorostiaga, J. (2019). Acceso y permanencia en universidades del Conurbano: logros y límites de las políticas institucionales. En A. M. Ezcurra (Coord.), Derecho a la educación. Expansión y desigualdad: tendencias y políticas en Argentina y América Latina (pp. 71-83). Universidad de Tres de Febrero.

Chen, X., \& Carroll, C. D. (2005). First-Generation Students in Postsecondary Education. A Look at Their College Transcripts. Postsecondary Education Descriptive Analysis Report. U.S. Department of Education Institute of Education Sciences NCES. https://nces.ed.gov/pubs2005/2005171.pdf 
Chesters, J. (2015). Maintaining inequality despite expansion: evidence of the link between parents' education and qualitative differences in educational attainment. Higher Education Quaterly, 69(2), 138-157. https://doi.org/10.1111/hequ.12060

Chiroleu, A. (2014). Alcances de la democratización universitaria en América Latina. Revista Iberoamericana De Educación, 65(1), 1-14. https://doi.org/10.35362/rie651328

Choy, S. P. (2001). Students Whose Parents Did Not Go to College: Postsecondary Access, Persistence, and Attainment National Center for Education Statistics. U.S. Department of Education, Office of Educational Research and Improvement. https://nces.ed.gov/pubs2001/2001126.pdf

Ezcurra, A. M. (2019). Educación superior: una masificación que incluye y desiguala. En A. M. Ezcurra (Coord.), Derecho a la educación. Expansión y desigualdad: tendencias y políticas en Argentina y América Latina (pp. 21-52). Universidad de Tres de Febrero.

Ezcurra, A. M. (2020a). Democratización y desigualdades. El ciclo superior en América Latina en el siglo XXI. Notas preliminares. En F. Acevedo (Coord.), Expansión de la educación superior en América Latina (pp. 121-144). Universidad de la República.

Ezcurra, A. M. (2020b). Educación Superior en el Siglo XXI. Una democratización paradojal. Escenarios globales y latinoamericanos. Revista Latinoamericana de Políticas y Administración de la Educación, 12(7), 112-127. https://revistas.untref.edu.ar/index.php/relapae/article/view/449/532

Fernández Lamarra, N., Pérez Centeno, C., Marquina, M., \& Aiello, M. (Eds.). (2018). Educación superior universitaria Argentina. Situación actual en el contexto regional. Universidad Nacional de Tres de Febrero.

García de Fanelli, A. (2019). Panorama de la Educación Superior en Iberoamérica. Red indicES. http://www.redindices.org/novedades/84-panorama-de-la-educacion-superior-en-iberoamerica-a-traves-delos-indicadores-de-la-red-indices

García de Fanelli, A. (en prensa). Políticas para promover el acceso con equidad en la educación superior latinoamericana. Serie Análisis Comparativos de Políticas Educativas. IIPE-UNESCO.

García de Fanelli, A. M., \& Balán, J. (1994). Expansión de la oferta universitaria: nuevas instituciones, nuevos programas. Documentos CEDES, Serie Educación Superior N. ${ }^{\circ} 106$. https://repositorio.cedes.org/handle/123456789/3409

García de Fanelli, A., \& Jacinto, C. (2010). Equidad y educación superior en América Latina. El papel de las carreras terciarias y universitarias. Revista Iberoamericana de Educación Superior, 1(1), 58-75.

Gasparini, L. (2002). On the Measurement of Unfairness: an application to high-school attendance in Argentina. Social Choice and Welfare, 19, 795-810. https://doi.org/10.1007/s003550200156

Gilardi, S., \& Guglielmetti, C. (2011). University life of non-traditional students: Engagement styles and impact on attrition. The Journal of Higher Education, 82(1), 33-53.

Groisman, F. (2016). Estructura social e informalidad laboral en Argentina. EUDEBA.

Haimovich Paz, F. (2017). Equity, Quality, and Variety of Higher Education. En M. M. Ferreyra, C. Avitabile, J. Botero Álvarez, F. Haimovich Paz, \& S. Urzúa, At a Crossroads. Higher Education in Latin America and the Caribbean (pp. 77-113). Work Bank Group. https://doi.org/10.1596/978-1-4648-1014-5_ch2 
Hass, C., \& Hadjar, A. (2020). Students' trajectories through higher education: a review of quantitative research. Higher Education, 79, 1099-1118. https://doi.org/10.1007/s10734-019-00458-5

Kisilevsky, M. (2002). Condiciones sociales y pedagógicas de ingreso a la educación superior en la Argentina. En M. Kisilevsky, \& C. Veleda, Dos estudios sobre el acceso a la educación superior en la Argentina (pp. 14-81). IIPE-UNESCO Sede Regional Buenos Aires.

Levy Yeyati, E., Favatta, F., Montane, M., \& Schteingart, D. (2018). Radiografía del trabajo argentino. School of Government Working Papers 201801. https://ideas.repec.org/p/udt/wpgobi/201801.html

Lucas, S. R. (2001). Effectively Maintained Inequality: Education Transitions, Track Mobility and Social Background Effects. American Journal of Sociology,106(6), 1642-1690. https://doi.org/10.1086/321300

Lucas, S. R. (2017). An archaeology of effectively maintained inequality theory. American Behavioral Scientist, 61(1), 8-29. https://doi.org/10.1177/0002764216682989

Marginson, S. (2016). High Participation Systems of Higher Education. The Journal of Higher Education, 87(2), 243-271. https://doi.org/10.1080/00221546.2016.11777401

Marquina, M. (2011). El ingreso a la universidad a partir de la reforma de los 90: las nuevas universidades del conurbano bonaerense. En N. Gluz (Comp.), Admisión a la universidad y selectividad social (pp. 63-86). Universidad Nacional de General Sarmiento.

OECD. (2018). Equity in Education: Breaking Down Barriers to Social Mobility. OECD Publishing. https://doi.org/10.1787/9789264073234-en

OECD. (2019). How does socio-economic status influence entry into tertiary education? Education Indicators in Focus 69. https://doi.org/10.1787/22267077

Pascarella, E.T., Pierson, C.T., Wolniak, G.C., \& Terenzini, P. T. (2004). First-Generation College Students. The Journal of Higher Education, 75(3), 249-284. https://doi.org/10.1080/00221546.2004.11772256

Paz, J. A., \& Cid, J. C. (2012). Determinantes de la asistencia escolar de los jóvenes en la Argentina. Revista Electrónica de Investigación Educativa, 14(1), 136-152.

Rabossi, M. (2011). The Private University Sector in Argentina: A Limited and Selective Expansion. Excellence in Higher Education, 2(1), 42-50. https://doi.org/10.5195/ehe.2011.28

Raftery, A. E., \& Hout, M. (1993). Maximally maintained inequality: Expansion, reform, and opportunity in Irish education, 1921-75. Sociology of Education, 66, 41-62. https://doi.org/10.2307/2112784

Troiano, H., Torrents, D., \& Daza, L. (2019). Compensation for poor performance through social background in tertiary education choices. Studies in Higher Education, 46(6), 1-17. https://doi.org/10.1080/03075079.2019.1666262

Trombetta, A. (2000). Los estudiantes terciarios: sus características socioculturales y sus motivaciones en la elección de una carrera. CEDES. https://repositorio.cedes.org/bitstream/123456789/3938/1/5175.pdf

Trow, M. (2006). Reflection on the transition from elite to mass to universal access: forms and phases of higher education in modern societies since WWII. En J. J. F. Forest \& P. G. Altbach, International Handbook of Higher Education (pp. 243-280). Springer. https://doi.org/10.1007/978-1-4020-4012-2_13 
Valdés Fernández, M. (2019). Efectos primarios y secundarios en la expectativa de matriculación universitaria: la desigualdad como reto del siglo XXI. Revista Prisma Social, (25), 332-358. https://revistaprismasocial.es/article/view/2595

Veleda, C. (2002). Estrategias individuales y familiares en la elección de las instituciones de educación superior. En M. Kisilevsky, \& C. Veleda, C. Dos estudios sobre el acceso a la educación superior en la Argentina (pp. 89-139). IIPE-UNESCO. https://www.buenosaires.iiep.unesco.org/es/publicaciones/dos-estudios-sobre-elacceso-la-educacion-superior-en-la-argentina

Vieira do Nascimento, D., Mutize,T., \& Roser Chinchilla, J. F. (2020). Hacia el acceso universal a la educación superior: tendencias internacionales. https://www.iesalc.unesco.org/2020/11/17/el-iesalc-lanza-el-informehacia-el-acceso-universal-a-la-educacion-superior-tendencias-internacionales/

\section{Cómo citar}

Adrogué, C., \& García de Fanelli, A. (2021). Brechas de equidad en el acceso a la educación superior argentina. Páginas de Educación, 14(2), 28-51. https://doi.org/10.22235/pe.v14i2.2507

\section{Contribución autoral}

a) Concepción y diseño del trabajo; b) Adquisición de datos; c) Análisis e interpretación de datos; d) Redacción del manuscrito; e) revisión crítica del manuscrito.

C. A. ha contribuido en b, c, d, e; A. G. d. F. en a, c, d, e.

Editora científica responsable: Mag. Florencia de León 